\title{
Intra-articular injection of orthobiologics in patients undergoing high tibial osteotomy for knee osteoarthritis is safe and effective - a systematic review
}

\author{
Brjan Kaiji Betzler ${ }^{1}$, Aiman Haziq Bin Muhammad Ridzwan Chew ${ }^{2}$ and Hamid Rahmatullah Bin Abd Razak B, $^{3}$ (I)
}

\begin{abstract}
Purpose: To qualitatively evaluate the current evidence reporting outcomes of intra-articular injection of orthobiologics in patients undergoing high tibial osteotomy (HTO) for osteoarthritis of the knee.

Methods: A systematic search methodology of the PUBMED, EMBASE, and CINAHL databases was conducted in July 2021. The search workflow was in adherence to the Preferred Reporting Items for Systematic Reviews and Meta-Analyses (PRISMA). The following inclusion criteria were adopted: clinical trials of any level of evidence, reporting outcomes following intra-articular injection of orthobiologics during high tibial osteotomy for knee osteoarthritis, with a minimum number of 10 patients treated. Duplicate data, studies on implanted orthobiologics and articles not written in English were excluded from this review.

Results: Eight studies were included in this review, with a total of 585 patients. Outcomes were discussed based on the types of orthobiologics used: (i) Platelet-Rich Plasma (PRP), (ii) Bone Marrow Aspirate Concentrate (BMAC), and (iii) Injected Mesenchymal Stem Cells (MSCs). Two studies utilised PRP, 4 studies utilised BMAC and 4 studies utilised injected MSCs.. Three studies provided Level II evidence and five studies provided Level III evidence. Statistically significant improvements in outcomes were documented in multiple trials, with few patients experiencing adverse events.

Conclusion: Intra-articular injection of orthobiologics in patients undergoing HTO is safe and effective with good outcomes reported. Due to the lack of high-level evidence, further research is required before this can be considered standard of care.
\end{abstract}

Level of evidence: III

Keywords: Osteotomy, Cartilage repair, Knee, Biologics, Osteoarthritis

\section{Introduction}

Osteoarthritis (OA) is a degenerative bone disease characterised by loss of cartilage, bone remodelling in the adjacent bone structures, and inflammation of surrounding tissues [1]. Globally, it is the most prevalent

\footnotetext{
*Correspondence: hamidrazak@gmail.com

${ }^{3}$ Department of Orthopaedic Surgery, Sengkang General Hospital, 110

Sengkang East Way, Singapore 544886, Singapore

Full list of author information is available at the end of the article
}

degenerative joint disease [2], and the most common cause of knee pain. Deformities seen in knee OA such as genu varum further worsens function by altering the mechanical axis of the lower limb, placing additional stress on the arthritic medial compartment. Treatment modalities of OA to date have primarily focused on reducing the rate of cartilage degeneration. However, newer techniques have evolved, focusing on increasing the rate of cartilage regeneration. 
High tibial osteotomy (HTO) is an effective procedure in the management of medial compartment knee OA with varus deformity, in young or physically active patients $[3,4]$. It corrects the mechanical axis of the knee, reducing the rate of cartilage degeneration by improving weight distribution within the knee joint $[5,6]$. Besides improved outcomes, several studies have also reported cartilage regeneration [7-10]. Concurrent procedures, such as the injection of orthobiologics during a HTO, have shown promise in enhancing cartilage regeneration in knee OA.

Orthobiologics are a relatively new treatment modality that has gained popularity recently due to its minimally invasive nature, and the potential for healing and recovery [11]. Broadly, orthobiologics include platelet rich plasma (PRP), plasma rich in growth factors (PRGF), bone marrow aspirate concentrate (BMAC) and mesenchymal stem cells (MSC). These products have the potential to aid in regeneration and recovery of cartilage [12]. While PRP and PRGF are rich in growth factors, BMAC and MSC both contain stem cells, with efficacy depending on multiple factors including source, proliferation capacity, and concentration of growth factors. It is important to note that PRP and BMAC are considered point of care treatment modalities, whereas MSCs typically require expansion prior to injection. Recent studies have reported on the efficacy of these orthobiologic agents. They have shown to enhance the quality of cartilage regeneration which in turn has contributed to better clinical outcomes following HTO [6, 10, 13-15].

Despite promising literature on the intra-articular injection of orthobiologics during HTOs, there is at present no consensus if orthobiologics should be routinely used in HTOs. The aim of this study is to qualitatively evaluate the current evidence reporting outcomes of intra-articular injection of orthobiologics in patients undergoing HTO for OA of the knee.

\section{Methods}

\section{Information sources and selection of studies}

An electronic search was performed by two independent authors (B.B. and A.H.) in the PUBMED, EMBASE, and CINAHL databases to identify all relevant studies published up to 10 July 2021. The search string used to query citation titles and abstracts was as follows: (Knee) AND (Osteotomy) AND (Biologics OR blood products OR PRP OR BMAC OR MSC OR Orthobiologics OR (Adipose derived OR Adipose derived mesenchymal stem cell OR synovial mesenchymal stem cell OR bone marrow mesenchymal stem cell) OR hUCB OR allogenic products OR amniotic fluid OR autologous conditioned serum OR stromal vascular fraction OR microfragmented adipose tissue OR PRGF OR amniotic membrane)". This review was not registered on the PROSPERO database. The search workflow was in adherence to the Preferred Reporting Items for Systematic Reviews and Meta-Analyses (PRISMA) [16], and is showcased in Fig. 1.

To identify studies to be included in the final review, the articles were independently assessed by two authors, B.B. and A.H., to determine eligibility for inclusion in the analysis. Any disagreements were resolved by consensus discussion among the authors. A total of eight studies were included in the final review.

\section{Eligibility criteria}

We included clinical trials of any level of evidence, reporting outcomes following HTO and concurrent injection of orthobiologics, including mesenchymal stem cells (MSCs),platelet-rich plasma (PRP), plasma rich in growth factors (PRGF), amniotic products, adiposederived products, bone marrow aspirate concentrate (BMAC) or autologous conditioned serum with a minimum number of 10 patients treated. Case reports, review articles, published abstracts, studies involving less than 10 patients, and duplicate data (the most recent series was included) were excluded from this review. Studies which evaluated only implanted orthobiologics (including implanted MSCs) were excluded because they are considered reparative procedures and outcomes would be expected to be significantly different as compared to injected orthobiologics. Studies which compared implanted with injected orthobiologics were included for their data on the injected orthobiologics. Articles not written in English, or where access to the full text was unavailable, were also excluded.

\section{Data collection and statistical analysis}

A total of 312 records were identified, of which 227 remained after removal of duplicates. Following Title and Abstract Screening, 23 Articles were identified and assessed in full text screening. Seven articles were then excluded because of their assessment of bone growth rather than cartilage repair, with a further eight articles excluded due to high fibular osteotomy (HFO) procedure instead of HTO, implantation of MSCs and lack of quantitative data.

All data from the texts, figures, and tables of the included studies were extracted to Microsoft Excel spreadsheet software for analysis and review. The specific information extracted included the following: (1) study details, including study design and level of evidence, (2) study population details, including number of patients, the size of the control group (if any), and the surgical procedures performed, (3) objective of study (4) intervention instituted, (5) Biologics system used and composition and quality of PRP (if PRP 
312 citations identified through database searching (Pubmed, EMBASE, CINAHL)

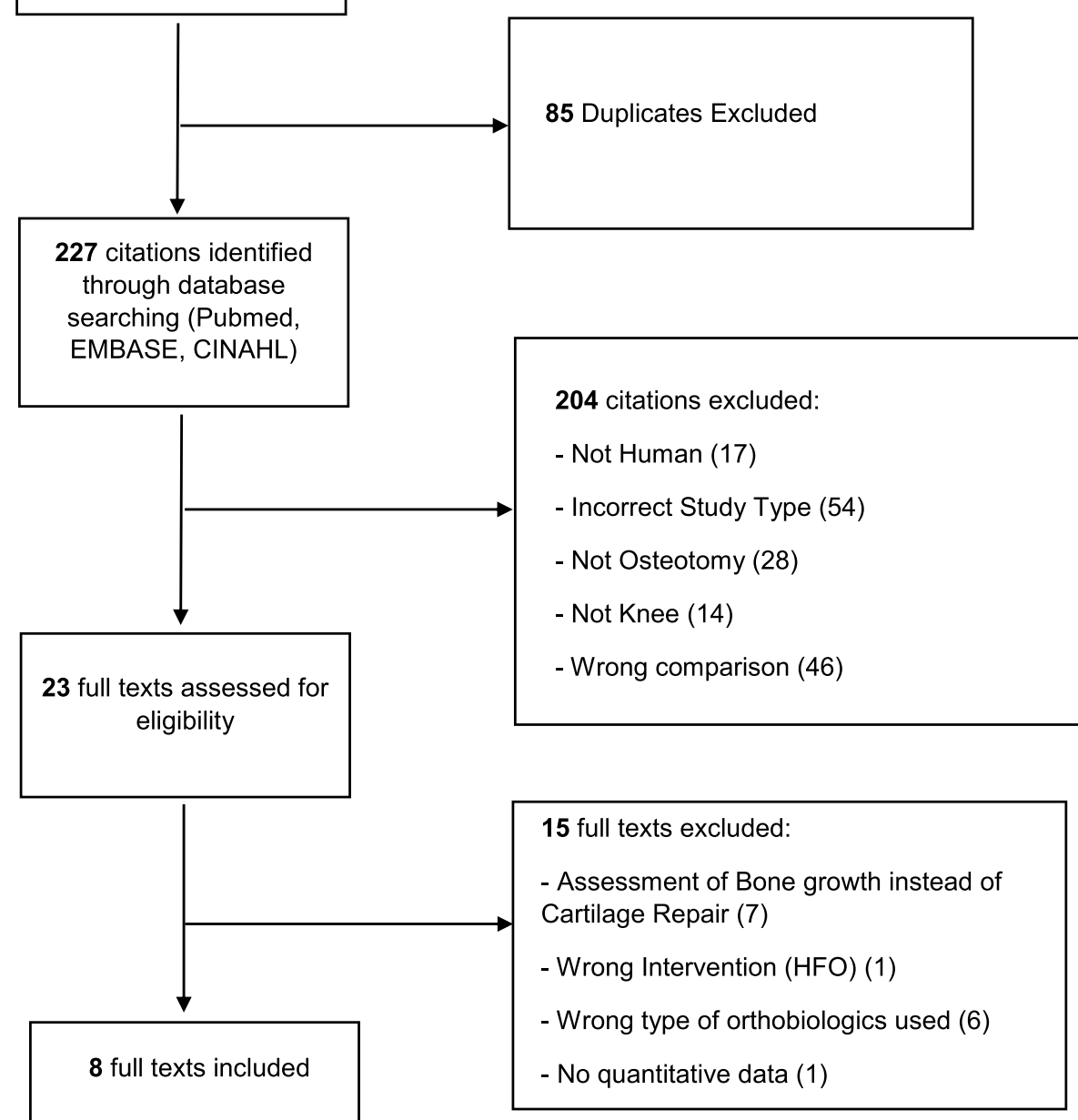

Fig. 1 PRISMA Flowchart. The search workflow was performed in accordance to the Preferred Reporting Items for Systematic Reviews and Meta-Analyses (PRISMA)

was used) (6) outcomes studied and criteria/scores used to quantify them and (7) results and any reported complications.

\section{Quality assessment of studies}

The quality of the Randomised Controlled Trials (RCT) included in this study was assessed using the Cochrane Collaboration risk assessment tool [17] while non-randomized studies were assessed using the Risk of Bias in Non-Randomised Studies - of Intervention (ROBINSI) tool [18]. The results of the Quality Assessment are detailed in Table 1.

\section{Results}

The eight studies $[15,19-25]$ included in this systematic review included a total of 585 patients. The results are presented according to the utilised orthobiologic agent as follows: two studies evaluated PRP, four evaluated injected culture-expanded MSCs, and four evaluated BMAC which were point-of-care unexpanded MSCs. Two studies included the use of dual orthobiologic agents $[19,21]$. For studies with patients that underwent second-look arthroscopy, these were conducted within a range of 1 to 2 years following index surgery. All other data was collected within a range of one to three-and-ahalf years post-procedure. With regards to study design, 
Table 1 Risk of bias in included studies

\begin{tabular}{|c|c|c|c|c|c|c|c|c|}
\hline RCTs & $\begin{array}{l}\text { Random } \\
\text { Sequence } \\
\text { Generation }\end{array}$ & $\begin{array}{l}\text { Allocation } \\
\text { Concealment }\end{array}$ & $\begin{array}{l}\text { Blinding of } \\
\text { participants } \\
\text { and person- } \\
\text { nel }\end{array}$ & $\begin{array}{l}\text { Blinding of } \\
\text { outcome } \\
\text { assessment }\end{array}$ & $\begin{array}{l}\text { Incomplete } \\
\text { outcome } \\
\text { data }\end{array}$ & $\begin{array}{l}\text { Selective } \\
\text { reporting }\end{array}$ & Other Bias & \\
\hline $\begin{array}{l}\text { D'Elia et al, } \\
\text { Revista Brasileira } \\
\text { de Ortopedia } \\
2015 \text { [19] }\end{array}$ & Low Risk & Low Risk & Unclear Risk & Unclear Risk & Low Risk & Unclear Risk & Unclear Risk & \\
\hline $\begin{array}{l}\text { Wong et al, } \\
\text { Arthroscopy } \\
2013 \text { [20] }\end{array}$ & Low Risk & Low Risk & Low Risk & Unclear Risk & Low Risk & Low Risk & Unclear Risk & \\
\hline $\begin{array}{l}\text { Koh et al, } \\
\text { Arthroscopy } \\
2014 \text { [21] }\end{array}$ & Low Risk & Low Risk & Low Risk & Unclear Risk & Low Risk & Low Risk & High Risk & \\
\hline Non- RCTs & Confounding & $\begin{array}{l}\text { Selection of } \\
\text { Participants }\end{array}$ & $\begin{array}{l}\text { Classifica- } \\
\text { tion of inter- } \\
\text { ventions }\end{array}$ & $\begin{array}{l}\text { Devia- } \\
\text { tions from } \\
\text { intended } \\
\text { interven- } \\
\text { tions }\end{array}$ & Missing Data & $\begin{array}{l}\text { Measurement } \\
\text { of outcomes }\end{array}$ & $\begin{array}{l}\text { Selection } \\
\text { of reported } \\
\text { results }\end{array}$ & $\begin{array}{l}\text { Overall ROB } \\
\text { judgements }\end{array}$ \\
\hline $\begin{array}{l}\text { Magnanelli et al, } \\
\text { Acta Biomedica } \\
2020[22]\end{array}$ & Moderate & Moderate & Low & Low & Low & Low & Low & Moderate \\
\hline $\begin{array}{l}\text { Kim et al, Ameri- } \\
\text { can Journal of } \\
\text { Sports Medicine } \\
2018 \text { [23] }\end{array}$ & Moderate & Moderate & Low & Low & Low & Low & Low & Moderate \\
\hline $\begin{array}{l}\text { Lee et al, } \\
\text { Arthroscopy: } \\
\text { The Journal of } \\
\text { Arthroscopic } \\
\text { and Related } \\
\text { Surgery } 2021 \\
\text { [24] }\end{array}$ & Moderate & Moderate & Low & Low & Low & Low & Low & Moderate \\
\hline $\begin{array}{l}\text { Jin et al, Knee } \\
\text { Surgery, Sports } \\
\text { Traumatology, } \\
\text { Arthroscopy } \\
2021 \text { [15] }\end{array}$ & Moderate & Moderate & Low & Low & Low & Low & Low & Moderate \\
\hline $\begin{array}{l}\text { Yang et al, Knee } \\
\text { Surgery, Sports } \\
\text { Traumatology, } \\
\text { Arthroscopy } \\
2021 \text { [25] }\end{array}$ & Moderate & Low & Low & Low & Low & Low & Low & Moderate \\
\hline
\end{tabular}

three $(37.5 \%)$ studies provided Level II evidence and five (62.5\%) studies provided Level III evidence. Characteristics of the studies are summarized in Table 2.

\section{Scoring systems utilized}

Multiple evaluation tools were utilized in the eight studies. The criteria, grading and descriptions of the systems discussed are listed here.

The ICRS-CRA score [26] has three components of evaluation: (i) degree of defect repair, (ii) integration to border zone, and (iii) macroscopic appearance. These components are graded normal (Grade I), nearly normal (Grade II), abnormal (Grade III), and severely abnormal (Grade IV). All studies reported second-look arthroscopy being conducted at a minimum of 1 year duration postoperatively. Four of the eight studies [15, 23-25] utilised this system.

The Koshino Staging System evaluates the status of the regenerated cartilage according to the macroscopic staging system described by Koshino et al. [9]. The staging system grades the regenerated cartilage as follows: (i) no regenerative change (Stage A), (ii) pink fibrous tissue with or without partial coverage with white fibrocartilage (Stage B), (iii) total cartilage regeneration with white overgrown cartilage (Stage C-1), and (iv) total cartilage regeneration with white even smooth cartilage (Stage C-2). All studies reported second-look arthroscopy being conducted at a minimum of 1-year following 
Table 2 Summary of included studies

\begin{tabular}{|c|c|c|c|c|c|c|}
\hline Study & $\begin{array}{l}\text { Level of } \\
\text { Evidence }\end{array}$ & $\begin{array}{l}\text { Type of Osteotomy } \\
\text { Performed }\end{array}$ & Intervention & $\begin{array}{l}\text { Number of Patients } \\
\text { in Intervention } \\
\text { Group }\end{array}$ & $\begin{array}{l}\text { Number of } \\
\text { Patients in Control } \\
\text { Group }\end{array}$ & Complications \\
\hline $\begin{array}{l}\text { D'Elia et al, Revista } \\
\text { Brasileira de Ortope- } \\
\text { dia } 2015 \text { [19] }\end{array}$ & $\|$ & Opening Wedge HTO & PRP with BMAC & 11 & 14 & Nil reported \\
\hline $\begin{array}{l}\text { Lee et al, Arthros- } \\
\text { copy: The Journal } \\
\text { of Arthroscopic and } \\
\text { Related Surgery } \\
2021 \text { [24] }\end{array}$ & III & HTO & $\begin{array}{l}\text { Microfracture with } \\
\text { BMAC (42 patients) } \\
\text { Microfracture with } \\
\text { hUCB-MSC ( } 32 \\
\text { patients) }\end{array}$ & 74 & N.A & Nil reported \\
\hline $\begin{array}{l}\text { Jin et al, Knee Sur- } \\
\text { gery, Sports Trauma- } \\
\text { tology, Arthroscopy } \\
2021 \text { [15] }\end{array}$ & III & HTO & $\begin{array}{l}\text { Microfracture with } \\
\text { BMAC }\end{array}$ & 48 & 43 & Nil reported \\
\hline $\begin{array}{l}\text { Yang et al, Knee Sur- } \\
\text { gery, Sports Trauma- } \\
\text { tology, Arthroscopy } \\
2021 \text { [25] }\end{array}$ & III & HTO & $\begin{array}{l}\text { BMAC (55 Patients) } \\
\text { hUCB-MSCs ( } 55 \\
\text { Patients) }\end{array}$ & 110 & N.A & $\begin{array}{l}\text { BMAC: one patient } \\
\text { complained of } \\
\text { postoperative } \\
\text { stiffness }\end{array}$ \\
\hline $\begin{array}{l}\text { Kim et al, American } \\
\text { Journal of Sports } \\
\text { Medicine } 2018 \text { [23] }\end{array}$ & III & HTO & MSCs & 50 & 50 & Nil reported \\
\hline $\begin{array}{l}\text { Magnanelli et al, } \\
\text { Acta Biomedica } \\
2020 \text { [22] }\end{array}$ & III & HTO & $\begin{array}{l}\text { Autologous adipose } \\
\text { derived stem cells }\end{array}$ & 42 & 43 & Nil reported \\
\hline $\begin{array}{l}\text { Koh et al, Arthros- } \\
\text { copy } 2014 \text { [21] }\end{array}$ & $\|$ & Opening Wedge HTO & PRP with MSCs & 21 & 23 & Nil reported \\
\hline $\begin{array}{l}\text { Wong et al, Arthros- } \\
\text { copy } 2013 \text { [20] }\end{array}$ & $\|$ & $\begin{array}{l}\text { Medial Opening } \\
\text { Wedge HTO }\end{array}$ & $\begin{array}{l}\text { Cultured MSCs with } \\
\text { Hyaluronic Acid }\end{array}$ & 28 & 28 & Nil reported \\
\hline
\end{tabular}

HTO High Tibial Osteotomy, PRP Platelet-Rich Plasma, MSCs Mesenchymal Stem Cells, hUCB-MSCs Human Umbilical Cord Blood-Derived Mesenchymal Stem Cells, $B M A C$ Bone Marrow Aspirate Concentrate

index surgery. Two of the eight $[15,25]$ studies utilised this system.

The International Knee Documentation Committee (IKDC) Questionnaire [8] is a subjective scale that provides patients with an overall function score. Consisting of three categories, (i) symptoms, (ii) sports activity, and (iii) knee function, it provides a means of assessing postoperative clinical and functional outcomes of procedures on the knee. Irrgang et al. [27] previously reported that the Minimum Clinically Important Difference (MCID) for IKDC following cartilage restoration procedures was 9.8. This was met by the five studies that reported IKDC as an outcome [15, 20, 22, 23, 25].

The Knee Injury and Osteoarthritis Outcome (KOOS) score [28] is a subjective questionnaire that assesses long and short-term impact on the patient post knee injury. It consists of five categories (i) pain, (ii) symptoms, (iii) activities of daily living, (iv) sport and recreation function and (v) quality of life relating to the knee. It is used to assess the course of the knee injury and outcome of treatments. Three of eight studies [21, $22,25]$ utilised this system.

The Lysholm Knee Scoring System [29] is a patientreported system used to assess a patients' knee-specific symptoms. It consists of eight categories (i) pain, (ii) instability, (iii) locking, (iv) swelling, (v) limp, (vi) stair climbing, (vii) squatting, and (viii) need for support. Four of eight studies [20-23] utilised this scoring system.

The Western Ontario and McMaster Universities Arthritis (WOMAC) Index [30] is a self-administered questionnaire used to assess $\mathrm{OA}$ in the hip or knee. It consists of three categories (i) pain, (ii) stiffness and (iii) physical function. The MCID for WOMAC has been reported to be 15.0 [24]. This was met by the two studies that utilized the WOMAC index as an outcome [15, 24].

The Visual Analog Scale (VAS) [31] is a subjective single-item scale used to evaluate the pain intensity experienced by the patient. Two of eight $[19,21]$ studies utilised this scale.

The Tegner Activity Scale [29] is a single-item scale used to assess level of activity based on work and sports pre and post injury. Three of eight studies $[20,22,25]$ utilised this scale.

The Knee Society Score (KSS) [32] is used to assess the patients' knee and functional outcomes before and after treatment. It consists of two categories, pain and function. The MCID for the KSS pain category and 
function scores has been reported to be 3.0 and 5.6 respectively [24]. These were met by the two studies that utilized the KSS pain and function scores as outcome measures [15, 24].

\section{PRP studies}

Two studies $[19,21]$ evaluated PRP combined with high tibial osteotomy. The results of these studies are summarised in Table 3. D'Elia et al. [19] reported outcomes assessed with post-operative VAS in patients who underwent HTO with PRP and BMAC versus iliac bone graft. There was no significant difference between the groups $(p=0.538)$.

Koh et al. [21] reported outcomes in patients who underwent HTO with injection of PRP and adiposederived MSCs versus patients who underwent HTO with injection of PRP only. They reported the Lysholm score, VAS score and KOOS scoring system following surgery. There were no significant differences $(p=0.357)$ in the Lysholm score between the two groups. VAS score was significantly better in the group which received PRP in combination with adipose-derived MSCs $(p<0.001)$. Similarly, the KOOS pain subscale $(p<0.001)$ and symptoms subscale $(p<0.001)$ showed greater improvement in the group which received PRP in combination with adipose-derived MSCs.

\section{BMAC studies}

Four studies evaluated BMAC used in combination with HTO $[15,19,24,25]$. The results of these studies are summarised in Table 4. The results of D'Elia et al. [19] have been discussed in the PRP results section above.

Jin et al. [15] reported outcomes in patients who underwent HTO with BMAC augmentation against a control group of patients who underwent HTO with microfracture (MFx) alone. The results in this study were reported using the following scoring systems, ICRS-CRA, Koshino Staging System, WOMAC Index, IKDC, and the KSS pain and function score. There was a statistically significant $(p=0.035)$ improvement in the mean ICRS-CRA grade of the group that had the BMAC augmentation versus the group that had MFx alone. There were no significant differences $(p=0.187)$ found between the two groups with regards to the Koshino Staging System score. There were also no significant differences between the two groups when assessed with the WOMAC Index $(p=0.297)$, IKDC $(p=0.260)$, KSS pain $(p=0.136)$ and function $(p=0.445)$.

Yang et al. [25] reported outcomes in patients who underwent HTO with BMAC versus HTO with human umbilical cord blood-derived MSCs (hUCB-MSC). The results in this study were reported using the following scoring systems, ICRS-CRA, Koshino Staging System, IKDC, KOOS, and the Tegner Activity Scale.

With regards to ICRS-CRA, Yang et al. [25] reported a statistically significant $(p=0.040)$ difference between the two groups. In their study, the BMAC group achieved significantly improved clinical and macroscopic outcomes, but worse macroscopic outcomes against a comparison group of patients who underwent hUCB-MSC implantation. Outcomes assessed with the Koshino Staging System showed significantly $(p=0.057)$ better cartilage regeneration in the group who underwent HTO with hUCB-MSC implantation, versus the group who underwent $\mathrm{HTO}$ with BMAC augmentation. There were no significant differences reported between the scores obtained by the two groups at the final follow up for the IKDC $(p=0.092)$, Tegner Activity Scale $(p=0.858)$ and KOOS (all subcategories $p>0.05$ ).

Lee et al. [24] reported outcomes following HTO and MFx with BMAC versus HTO and MFx with hUCBMSC. The results in this study were reported using the following scoring systems, ICRS-CRA, WOMAC index, KSS pain and function score. Lee et al. [24] corroborated the findings of Yang et al. [25] with regards to the ICRSCRA score. The group that underwent BMAC augmentation showed significantly worse cartilage regeneration in both the medial femoral condyle $(p=0.001)$ and medial tibial condyle $(p=0.001)$ than the group that underwent hUCB-MSC implantation. There were no other significant differences between the two groups for the WOMAC Index $(p=0.080)$ and the KSS pain $(p=0.380)$ and function $(p=0.437)$ scores.

\section{Injected MSCs studies}

Four studies [20-23] reported outcomes following HTO and injected MSCs. The results of these studies are summarized in Table 5. The results reported by Koh et al. [21] were discussed in the PRP results section above. In all these studies, there was culture expansion of the MSCs.

Magnanelli et al. [22] evaluated the effect of adiposederived MSCs with HTO and compared this to a control group that underwent HTO alone. The results in this study were reported using the following systems, KOOS, IKDC, Lysholm Scoring system, and Tegner Activity Scale. For the KOOS system, significant $(P<0.05)$ improvement was found with regards to the activities of daily living category for the group treated with adipose derived MSCs. No significant differences were found in other categories of the KOOS system. No significant differences were found when using the IKDC, Lysholm Scoring System and the Tegner Activity Scale.

Kim et al. [23] compared outcomes between patients who underwent HTO with adipose-derived MSCs with a control group of patients who underwent HTO alone. 


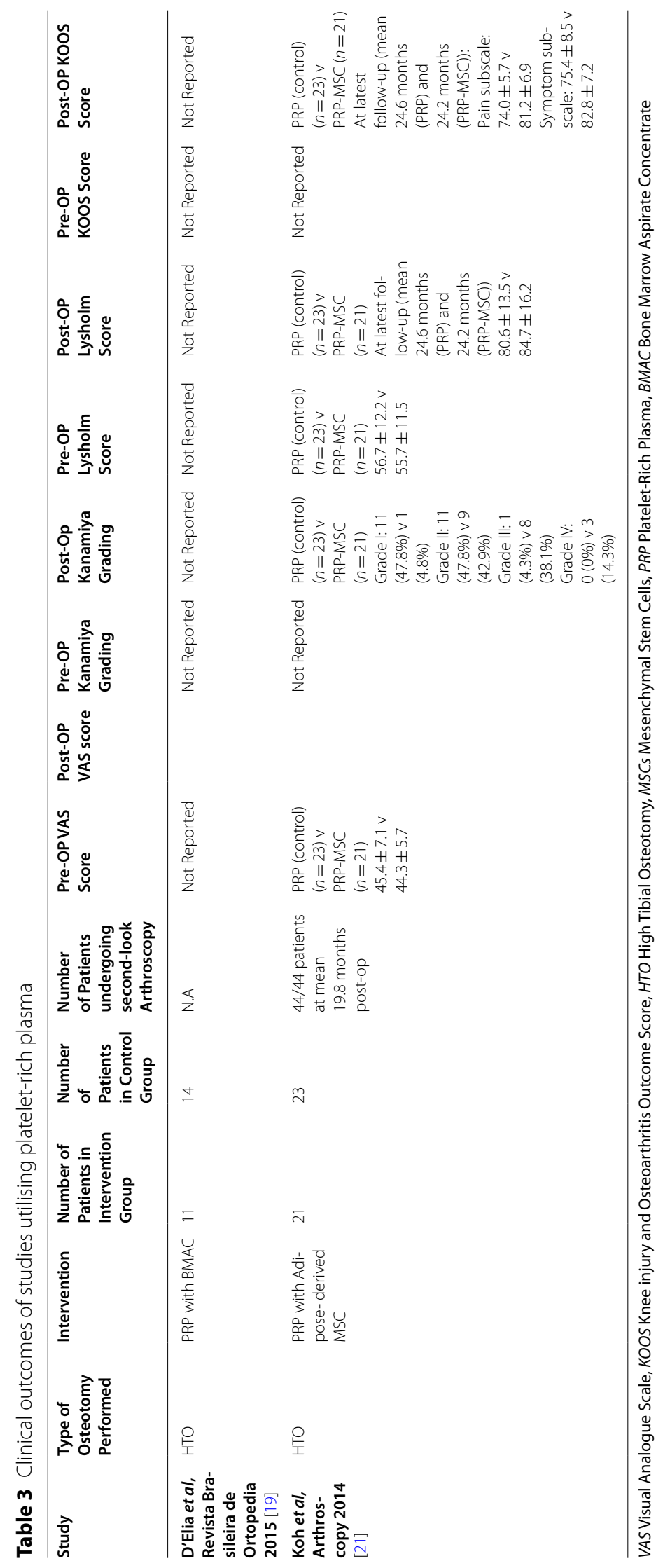




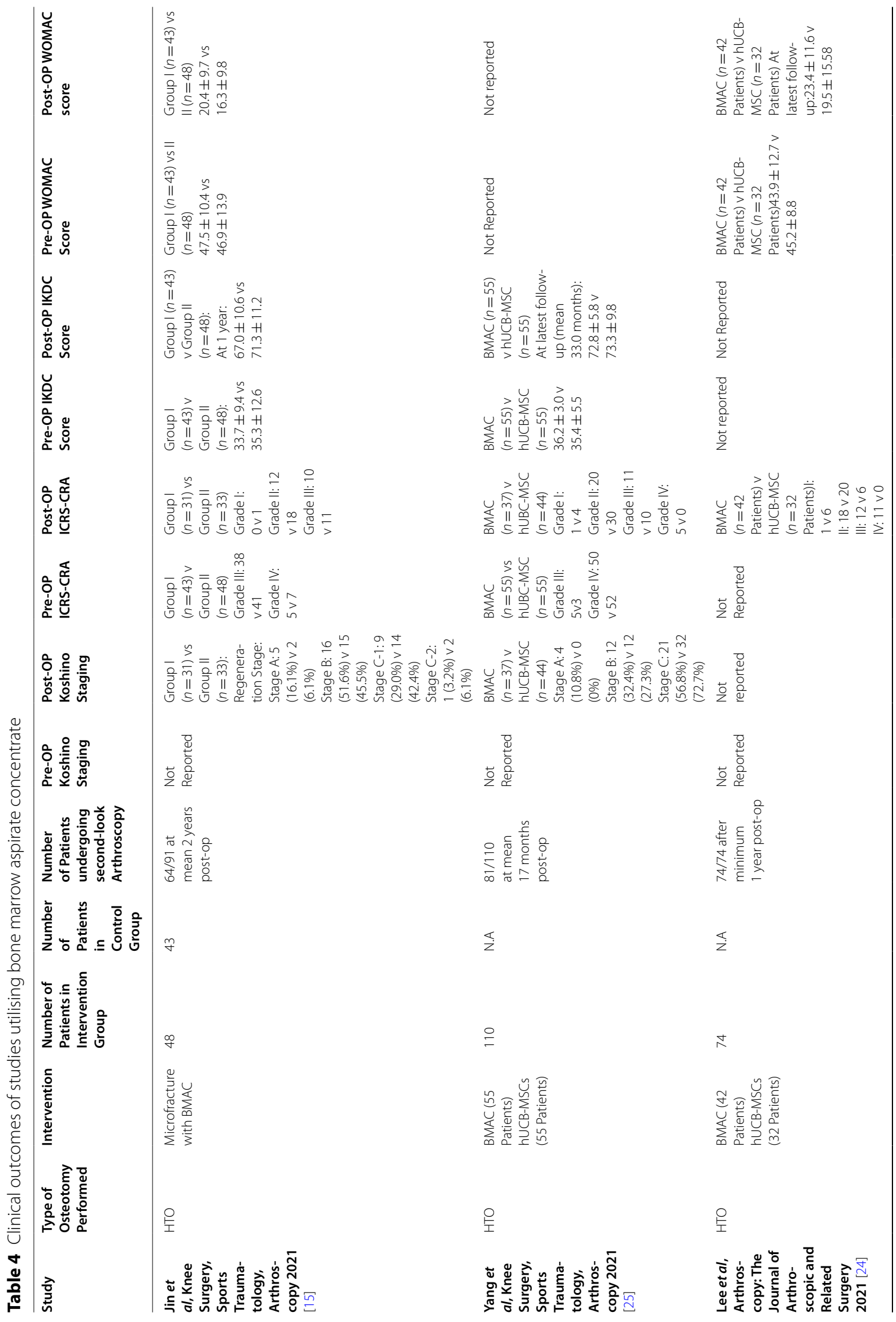




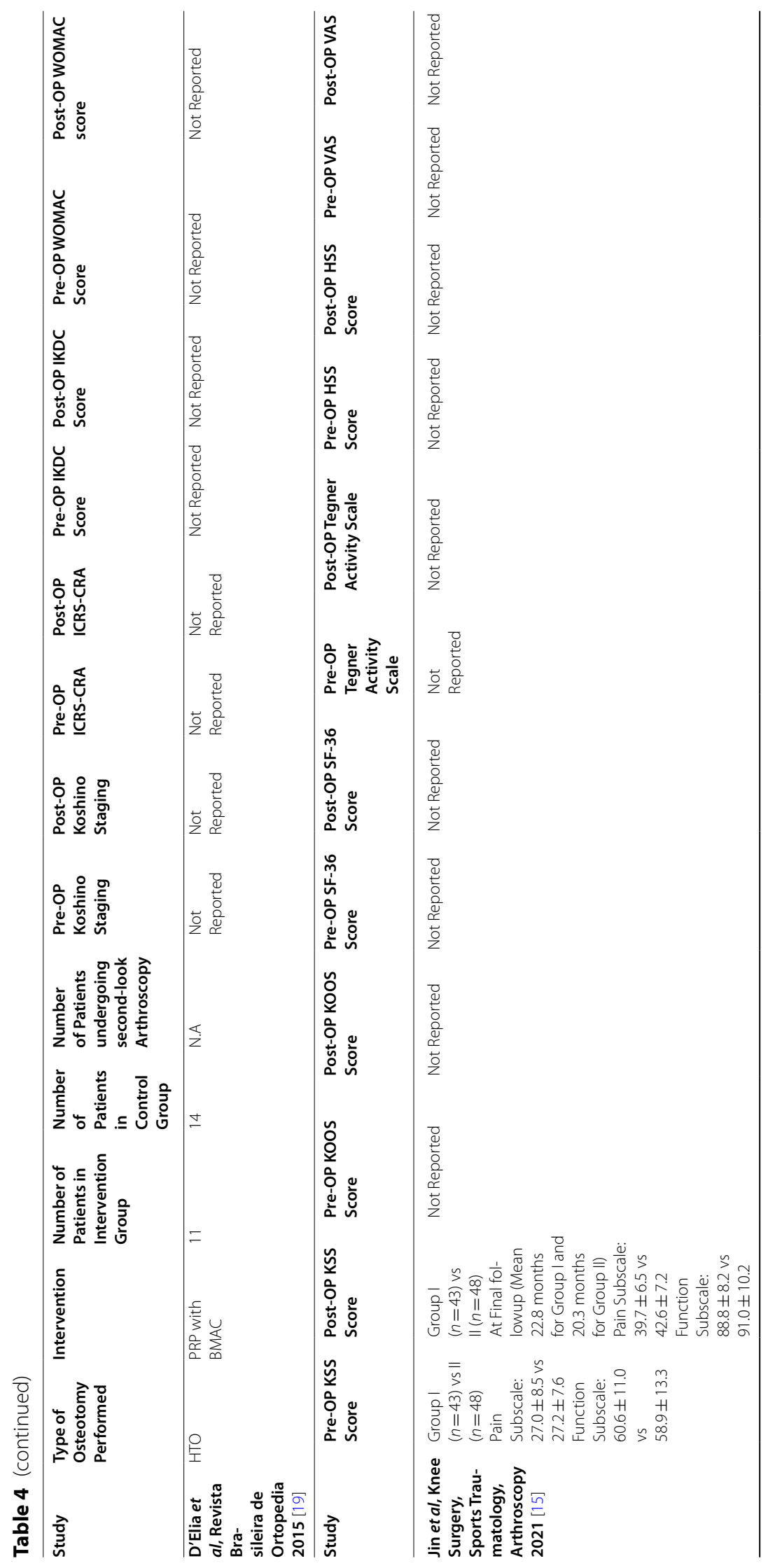




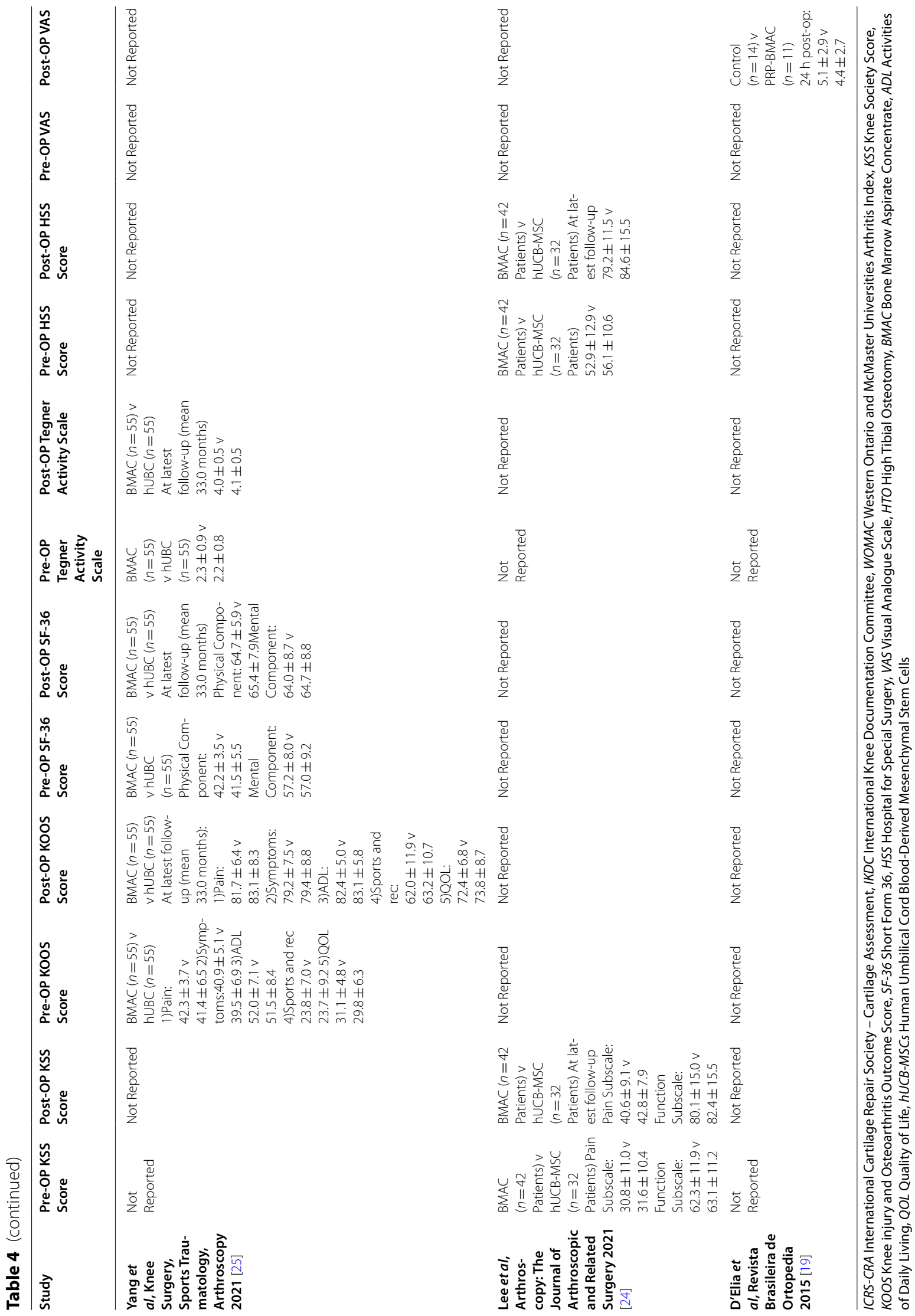




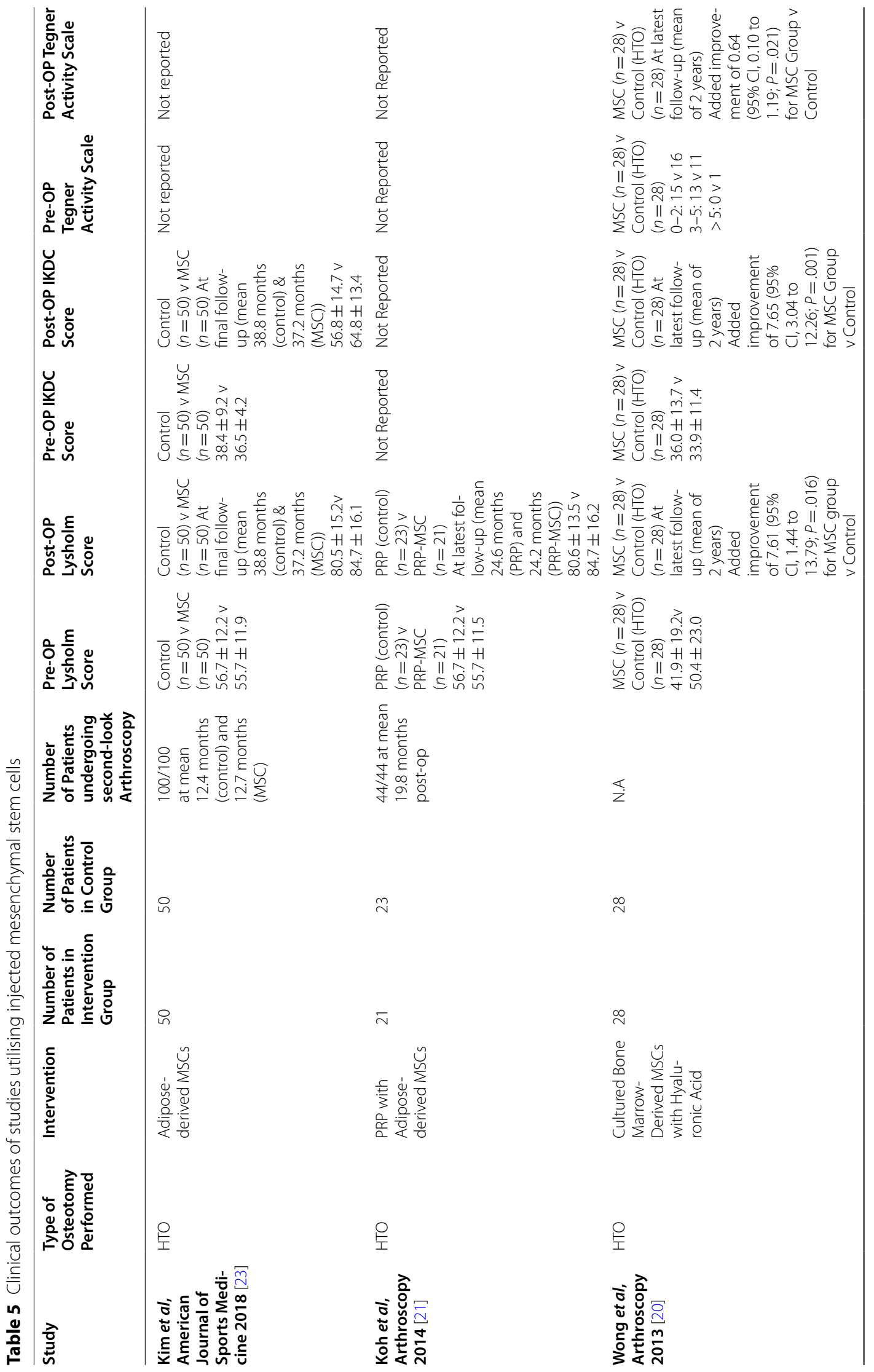




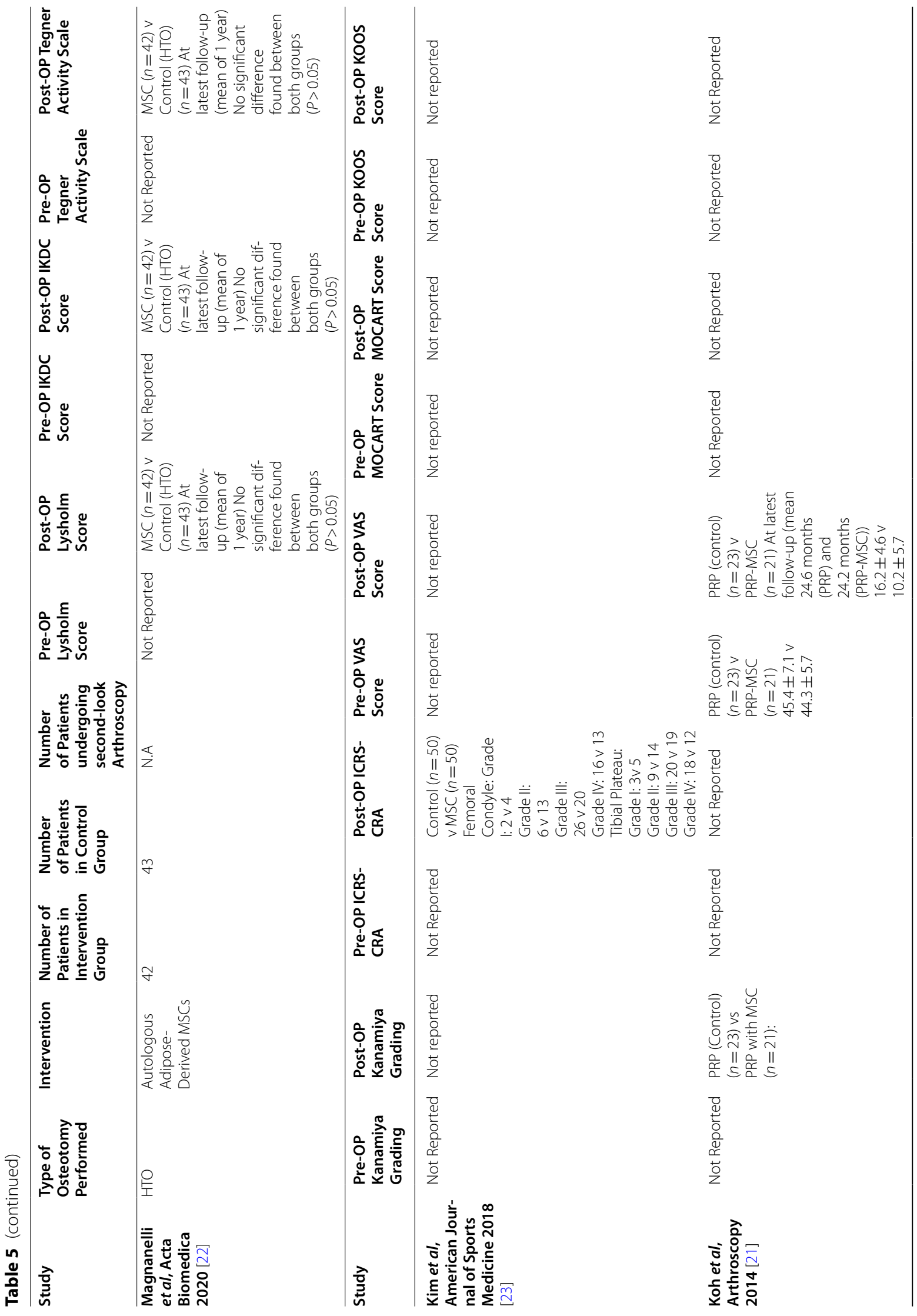




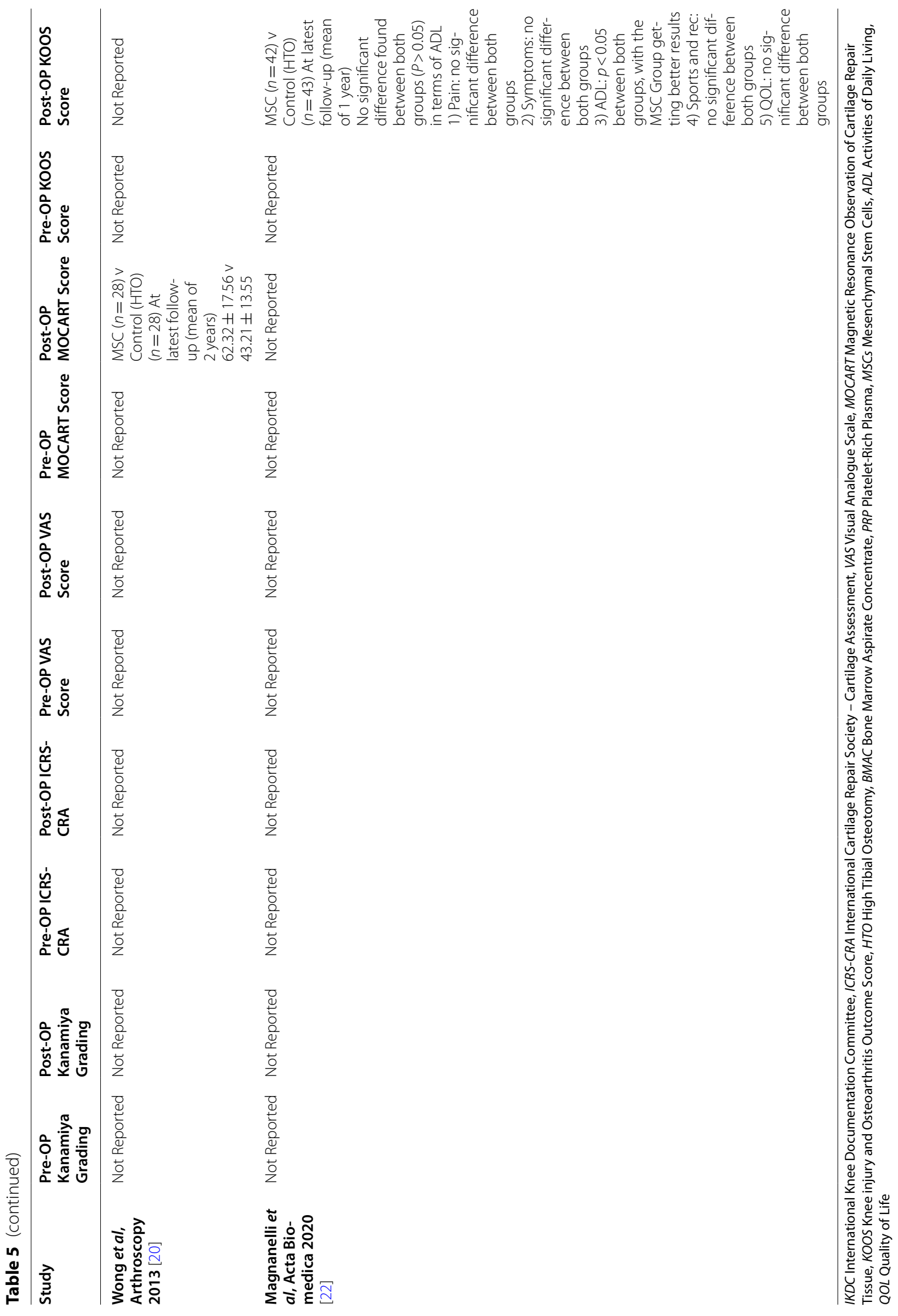


The results in this study were reported using the following systems, ICRS-CRA, IKDC, and Lysholm Scoring System. Unlike the results of Magnanelli et al. [22], Kim et al. [23] reported a statistically significant improvement in the mean ICRS-CRA grade of patients who underwent $\mathrm{HTO}$ with MSC injection with respect to cartilage regeneration at both the femoral condyle $(p=0.015)$ and the tibial plateau $(p=0.002)$. IKDC scores showed a significant $(p=0.049)$ difference in scores between the two groups, with the intervention group obtaining better scores at the final follow up post-operatively. There was also significant $(p=0.041)$ difference between the Lysholm scores between the two groups, with the group receiving adipose-derived MSCs obtaining better results.

Wong et al. [20] reported outcomes following HTO and injection of MSCs combined with hyaluronic acid versus HTO and injection of hyaluronic acid alone. The results in this study were reported using the following systems: IKDC, Lysholm Scoring system and Tegner Activity Scale. The authors reported a statistically better results in the group that underwent HTO and injection of MSCs combined with hyaluronic acid $(p=0.001)$ in terms of IKDC scores, supporting the findings of Kim et al. [23]. There was also significant differences $(p=0.016)$ between the two groups when using the Lysholm scoring system and the Tegner Activity Scale $(p=0.021)$ with the intervention group showing greater improvement than the control group, further supporting the findings of Kim et al. [23].

\section{Complications}

Out of 585 patients, there were no reports of severe postoperative complications nor any severe adverse reactions such as deep infections or failure of prosthesis implants. However, Yang et al. [25] reported one patient in the intervention group who underwent HTO with BMAC that complained of postoperative stiffness which selfresolved without the need of any follow-up procedures.

\section{Discussion}

This systematic review aimed to qualitatively evaluate the current evidence reporting outcomes of intra-articular injection of orthobiologics in patients undergoing HTO for OA of the knee. The key finding reported in this study is that there is a significant improvement in cartilage repair and regeneration following HTO when a concomitant injected orthobiologic product is used, except in studies when the injected orthobiologic is compared to an intervention utilising implanted MSC such as in the studies conducted by Yang et al. [25] and Lee et al. [24]. In our systematic review, we excluded implanted MSCs due to the nature of the procedure being reparative as compared to injected orthobiologics which are considered regenerative procedures. Thus, it is only fair that implanted MSCs and other reparative procedures be evaluated separately from injected orthobiologics as it would be expected that reparative procedures lead to far better macroscopic outcomes. Regardless, the absolute outcomes reported by Lee et al. [24] and Yang et al. [25] regarding injected MSCs remained acceptable when compared to other studies in this review. However, the authors do report discordance between macroscopic outcomes (ICRS-CRA, Koshino) and clinical findings (IKDC, KOOS, Lysholm, WOMAC, VAS, Tegner, KSS). Furthermore, due to the lack of high-level evidence, differing follow-up schedules, heterogeneity of intervention procedures between studies, and lack of a cost-benefit analysis, it is difficult to ascertain the true benefit that the various orthobiologic modalities provide when used concurrently with HTO. Studies with longer term follow-up are required to analyse if the increased quality of the repaired cartilage translates to functional and quality of life (QoL) improvements. Nonetheless based on our review, all the orthobiologics utilised in intervention groups have demonstrated good safety profiles and improvement in outcomes of cartilage repair. Hence, there is promise and potential for orthobiologics being used as an effective concomitant option for surgeons performing HTO [33].

Orthobiologic agents are believed to inhibit inflammatory processes and promote tissue healing [34]. Based on our results, all three agents such as PRP, BMAC and MSCs have largely been successful in improving outcomes following concomitant use with HTO. However, differences exist between the various orthobiologic agents based on the outcome measures, and the time frame within which the data was gathered. With regards to macroscopic outcomes, none of the papers that evaluated PRP presented data using ICRS-CRA or Koshino staging. Among the included studies reporting data on injected MSCs, Kim et al. [23] was the only study that reported ICRS-CRA, with significant improved outcomes in the intervention group, in line with significant clinical outcomes according to IKDC and Lysholm scoring. In contrast, BMAC studies present a mismatch between macroscopic and clinical outcomes, with three studies [15, 24, 25] reporting significant macroscopic but insignificant clinical outcomes. This can be attributed to high levels of heterogeneity between the papers which evaluated BMAC. Further minor reasons for this mismatch include differing MSC sources, different study designs with different interventions, and difference in follow-up times.

Based on the clinical outcome scores reported by Koh et al. [21], Kim et al. [23] and Wong et al. [20], the use of injected MSCs combined with another orthobiologic 
agent such as PRP or used on its own in a HTO procedure tends to produce a significantly better outcome in terms of cartilage regeneration and pain reduction if compared to HTO alone or if another orthobiologic agent was used on its own. MSCs are able to differentiate into chondrocytes as well as produce extracellular matrix molecules that are vital in cartilage regeneration and maintenance [35]. Thus the use of injected MSCs alongside other orthobiologics such as PRP tends to increase its efficacy due to its potential to promote the proliferation of MSCs as well as help to increase the ECM production [35], possibly contributing to the better outcomes as discussed above.

The study by Wong et al. [20] was the only one which presented data according to the Magnetic Resonance Observation of Cartilage Repair Tissue (MOCART) Knee Score [36], reporting significantly improved cartilage coverage of lesions with the usage of MSCs in HTO versus the control group of HTO with Hyaluronic Acid. This was accompanied by significantly better integration of the regenerated cartilage to the border zone with a lower rate of visible defects, with an age-adjusted mean difference in total MOCART score of 19.6. Despite this being the only included paper which presented MRIbacked data with regards to cartilage regeneration, the authors believe that the findings are significant given that MOCART is an objective score that provides a standardised, reproducible, and semiquantitative approach for the morphological assessment of cartilage repair [37]. Further studies which present MRI-backed data such as MOCART would be useful to discuss the balance between mechanics and biology in the pathogenesis and treatment of Knee OA.

In addition to knee-specific and joint-specific outcomes, another potential benefit of orthobiologics in HTO could be the reduction of postoperative blood loss, which remains a major complication of knee surgery. Perioperative and post-operative bleeding has been found to be associated with tourniquet use [12], alongside other bleeding risks involved in surgery. D'Elia et al. [19] reported on the change in haemoglobin $(\mathrm{Hb})$ and haematocrit (Hct) levels to evaluate the extent of blood loss $24 \mathrm{~h}$ postoperatively. No significant differences in the change in $\mathrm{Hb}$ and Hct levels $(p=0.820$ and $p=0.323$ respectively) pre- and postoperatively were reported. In current literature, several studies have reported the efficacy of PRP in reducing perioperative and postoperative bleeding. PRPs contain a high concentration of growth factors, thromboxane A2 and thrombin which would theoretically lead to more efficient platelet plug formation and haemostasis [38]. A meta-analysis done by $\mathrm{Ma}$ et al. [39] found that the use of PRP during total knee arthroplasty (TKA) significantly reduced total blood loss $(p=0.0005)$ and decreased $\mathrm{Hb}$ drop at post-operative day $1(p=0.008)$ when compared against a control group. Everts et al. [40] also reported similar results where the decline in $\mathrm{Hb}$ levels post-operative days one and two were significantly lower in the PRP group when compared against a control group ( $p<0.001$ and $p<0.01$ respectively). Therefore, PRP seems to exhibit a procoagulant effect, or at the very least may have a role in reducing perioperative and postoperative blood loss. However, due to conflicting findings and lack of high-level evidence, further high-level trials which also include relevant parameters such as prothrombin time are required to evaluate the efficacy of PRPs and other orthobiologics in reducing blood loss.

Finally, OA is a heterogeneous and multifactorial pathology and the underlying mechanisms causing the disease might differ between patients [41]. Given that HTO is indicated primarily in moderately active, highdemand, and relatively younger patients [42], the rate of conversion to TKA in these patients undergoing HTO with orthobiologics is a pertinent area of future research. The current literature is understandably limited in this area, given the relatively new status of orthobiologics as a concurrent treatment modality in HTO.

\section{Strengths and limitations}

In our search of the literature, Harris et al. [14] presented the only prior systematic review which explored the clinical outcomes of biologics on HTO. However, this analysis was based on the concomitant utilisation of articular cartilage surgery and/or meniscal allograft transplantation rather than orthobiologics. This current study is the first systematic review which attempts to evaluate clinical and macroscopic outcomes following HTO with concomitant use of orthobiologics. It adds to the literature by showing that patients achieved statistically significant improvement in outcomes following HTO with PRP, BMAC or injected MSCs. The heterogeneity of studies included in this review alludes to the fact that there is a need for more robust clinical trials with repeatable study designs across the spectrum of orthobiologics.

However, the findings discussed in this systematic review should be carefully considered in light of our limitations. Firstly, multiple studies lacked a comparison against a suitable control, thus the data was deemed insufficient for a meta-analysis to be carried out. Studies utilised different systems to assess cartilage healing and regeneration, resulting in the lack of a singular basis of comparison. Furthermore, significant improvements in cartilage healing and regeneration may not completely correlate to improvements in clinical and functional outcomes of the knee. This is pertinent given the known dissociation between radiographic signs and clinical symptoms in patients with osteoarthritis of the knee [43]. 
Despite some studies indicating the significant correlation between cartilage regeneration and clinical outcome $[15,19-21,23,25,44-47]$, more robust clinical trials are required to assess the degree to which this correlation can be established, in order to provide a holistic evaluation of the desired levels of cartilage regeneration that are associated with improvements in patient quality of life. An accurate assessment of financial costs of the multiple treatment regimes would also be required for a reliable cost-benefit analysis.

\section{Future research direction}

Based on our findings, there is a lack of high-level studies evaluating the effects of orthobiologic injections in conjunction with HTO. We hope that this systematic review will help lead the discussion, and encourage researchers to conduct more robust Level I and II clinical and translational studies. These would address factors and outcomes not discussed in this review such as, but not limited to, postoperative bleeding, cost-benefit analyses of treatment modalities, and other orthobiologic agents.

\section{Conclusion}

Intra-articular injection of orthobiologics in patients undergoing HTO is safe and effective with good outcomes reported. Due to the lack of high level of evidence, further research is required before this can be considered standard of care.

\begin{abstract}
Abbreviations
HTO: High tibial osteotomy; BMAC: Bone marrow aspirate concentrate; PRP: Platelet-rich plasma; MSCs: Mesenchymal stem cells; MFx: Microfracture; IKDC: International Knee Documentation Committee; MCID: Minimum Clinically Important Difference; ICRS-CRA: International Cartilage Repair Society Cartilage Assessment; VAS: Visual Analogue Scale; WOMAC: Western Ontario and McMaster Universities Arthritis Index; KSS: Knee Society Score; MOCART: Magnetic Resonance Observation of Cartilage Repair Tissue; KOOS: Knee injury and Osteoarthritis Outcome Score; SF-36: Short Form 36; ADL: Activities of daily living; QOL: Quality of life.
\end{abstract}

\section{Acknowledgements}

Not applicable.

\section{Authors' contributions}

All authors contributed to the conceptualisation, data extraction, and writing of this manuscript. All authors read and approved the final manuscript.

\section{Funding}

The authors declare no source of funding for this manuscript.

\section{Availability of data and materials}

Not applicable.

\section{Declarations}

Ethics approval and consent to participate Not applicable.
Consent for publication

Not applicable.

\section{Competing interests}

The authors declare no competing interests.

\section{Author details}

${ }^{1}$ Lee Kong Chian School of Medicine, Nanyang Technological University, 59 Nanyang Drive, Experimental Medicine Building, Singapore 636921, Singapore. ${ }^{2}$ Adelaide Medical School, Faculty of Health and Medical Sciences, 30 Frome Rd, Adelaide, SA 5000, Australia. ${ }^{3}$ Department of Orthopaedic Surgery, Sengkang General Hospital, 110 Sengkang East Way, Singapore 544886, Singapore. ${ }^{4}$ Sing Health Duke-NUS Musculoskeletal Sciences Academic Clinical Programme, 20 College Road, Academia Level 4, Singapore 169865, Singapore.

Received: 4 June 2021 Accepted: 17 August 2021

Published online: 27 September 2021

\section{References}

1. National Clinical Guideline Centre (UK) (2014) Osteoarthritis: care and management in adults. National Clinical Guideline Centre (UK), London

2. Chen D, Shen J, Zhao W, Wang T, Han L, Hamilton JL et al (2017) Osteoarthritis: toward a comprehensive understanding of pathological mechanism. Bone Res 5:16044

3. Amendola A, Bonasia DE (2010) Results of high tibial osteotomy: review of the literature. Int Orthop 34:155-160

4. Zuiderbaan HA, van der List JP, Kleeblad LJ, Appelboom P, Kort NP, Pearle AD et al (2016) Modern indications, results, and global trends in the use of unicompartmental knee arthroplasty and high tibial osteotomy in the treatment of isolated medial compartment osteoarthritis. Am J Orthop (Belle Mead NJ) 45:E355-E361

5. Kanamiya T, Naito M, Hara M, Yoshimura I (2002) The influences of biomechanical factors on cartilage regeneration after high tibial osteotomy for knees with medial compartment osteoarthritis: clinical and arthroscopic observations. Arthroscopy 18:725-729

6. Sterett WI, Steadman JR, Huang MJ, Matheny LM, Briggs KK (2010) Chondral resurfacing and high tibial osteotomy in the varus knee: survivorship analysis. Am J Sports Med 38:1420-1424

7. Jung WH, Takeuchi R, Chun CW, Lee JS, Ha JH, Kim JH et al (2014) Secondlook arthroscopic assessment of cartilage regeneration after medial opening-wedge high tibial osteotomy. Arthroscopy 30:72-79

8. Kahlenberg CA, Nwachukwu BU, Hamid KS, Steinhaus ME, Williams RJ 3rd (2017) Analysis of outcomes for high tibial osteotomies performed with cartilage restoration techniques. Arthroscopy 33:486-492

9. Koshino T, Wada S, Ara Y, Saito T (2003) Regeneration of degenerated articular cartilage after high tibial valgus osteotomy for medial compartmental osteoarthritis of the knee. Knee 10:229-236

10. Matsunaga D, Akizuki S, Takizawa T, Yamazaki I, Kuraishi J (2007) Repair of articular cartilage and clinical outcome after osteotomy with microfracture or abrasion arthroplasty for medial gonarthrosis. Knee 14:465-471

11. Chahla J, Mandelbaum BR (2018) Biological treatment for osteoarthritis of the knee: moving from bench to bedside-current practical concepts. Arthroscopy 34:1719-1729

12. Zlotnicki JP, Geeslin AG, Murray IR, Petrigliano FA, LaPrade RF, Mann BJ et al (2016) Biologic treatments for sports injuries II think tank-current concepts, future research, and barriers to advancement, part 3: articular cartilage. Orthop J Sports Med 4:2325967116642433

13. Cavallo M, Sayyed-Hosseinian SH, Parma A, Buda R, Mosca M, Giannini S (2018) Combination of high tibial osteotomy and autologous bone marrow derived cell implantation in early osteoarthritis of knee: a preliminary study. Arch Bone Jt Surg 6:112-118

14. Harris JD, McNeilan R, Siston RA, Flanigan DC (2013) Survival and clinical outcome of isolated high tibial osteotomy and combined biological knee reconstruction. Knee 20:154-161

15. Jin Q-H, Chung Y-W, Na S-M, Ahn H-W, Jung D-M, Seon J-K (2021) Bone marrow aspirate concentration provided better results in cartilage regeneration to microfracture in knee of osteoarthritic patients. Knee Surg Sports Traumatol Arthrosc 29:1090-1097 
16. Liberati A, Altman DG, Tetzlaff J, Mulrow C, Gotzsche PC, loannidis JP et al (2009) The PRISMA statement for reporting systematic reviews and metaanalyses of studies that evaluate healthcare interventions: explanation and elaboration. BMJ 339:b2700

17. Higgins JP, Altman DG, Gotzsche PC, Juni P, Moher D, Oxman AD et al (2011) The Cochrane Collaboration's tool for assessing risk of bias in randomised trials. BMJ 343:d5928

18. Sterne JA, Hernan MA, Reeves BC, Savovic J, Berkman ND, Viswanathan M et al (2016) ROBINS-l: a tool for assessing risk of bias in non-randomised studies of interventions. BMJ 355:14919

19. D'Elia CO, de Rezende MU, Bitar AC, Tatsui N, Pécora JR, Camanho GL (2015) The use of platelet rich plasma with bone marrow aspirate in puddu tibial osteotomy. Rev Bras Ortop 44:508-512

20. Wong KL, Lee KB, Tai BC, Law P, Lee EH, Hui JH (2013) Injectable cultured bone marrow-derived mesenchymal stem cells in varus knees with cartilage defects undergoing high tibial osteotomy: a prospective, randomized controlled clinical trial with 2 years' follow-up. Arthroscopy 29:2020-2028

21. Koh YG, Kwon OR, Kim YS, Choi YJ (2014) Comparative outcomes of open-wedge high tibial osteotomy with platelet-rich plasma alone or in combination with mesenchymal stem cell treatment: a prospective study. Arthroscopy 30:1453-1460

22. Magnanelli S, Screpis D, Di Benedetto P, Natali S, Causero A, Zorzi C (2020) Open-wedge high tibial osteotomy associated with lipogems ${ }^{\circledR}$ intra-articular injection for the treatment of varus knee osteoarthritis retrospective study. Acta Biomed 91:e2020022

23. Kim YS, Koh YG (2018) Comparative matched-pair analysis of openwedge high tibial osteotomy with versus without an injection of adipose-derived mesenchymal stem cells for varus knee osteoarthritis: clinical and second-look arthroscopic results. Am J Sports Med 46:2669-2677

24. Lee NH, Na SM, Ahn HW, Kang JK, Seon JK, Song EK (2021) Allogenic human umbilical cord blood-derived mesenchymal stem cells is more effective than bone marrow aspiration concentrate for cartilage regeneration after high tibial osteotomy in medial unicompartmental osteoarthritis of knee. Arthroscopy. https://doi.org/10.1016/j.arthro.2021. 02.022

25. Yang HY, Song EK, Kang SJ, Kwak WK, Kang JK, Seon JK (2021) Allogenic umbilical cord blood-derived mesenchymal stromal cell implantation was superior to bone marrow aspirate concentrate augmentation for cartilage regeneration despite similar clinical outcomes. Knee Surg Sports Traumatol Arthrosc. https://doi.org/10.1007/s00167-021-06450-w

26. Brittberg M, Peterson L (1998) Introduction of an articular cartilage classification. ICRS Newsletter 1:5-8

27. Irrgang JJ, Anderson AF, Boland AL, Harner CD, Kurosaka M, Neyret $P$, Richmond JC, Shelborne KD (2001) Development and Validation of the International Knee Documentation Committee Subjective Knee Form. Am J Sports Med 29(5):600-13. https://doi.org/10.1177/0363546501 0290051301

28. Roos EM, Lohmander LS (2003) The knee injury and osteoarthritis outcome score (KOOS): from joint injury to osteoarthritis. Health Qual Life Outcomes 1:64-64

29. Tegner Y, Lysholm J (1985) Rating systems in the evaluation of knee ligament injuries. Clin Orthop Relat Res 198:43-49

30. Bellamy N, Buchanan WW, Goldsmith CH, Campbell J, Stitt LW (1988) Validation study of WOMAC: a health status instrument for measuring clinically important patient relevant outcomes to antirheumatic drug therapy in patients with osteoarthritis of the hip or knee. J Rheumatol 15:1833-1840

31. McCormack HM, Horne DJ, Sheather S (1988) Clinical applications of visual analogue scales: a critical review. Psychol Med 18:1007-1019
32. Scuderi GR, Bourne RB, Noble PC, Benjamin JB, Lonner JH, Scott WN (2012) The new knee society knee scoring system. Clin Orthop Relat Res 470:3-19

33. Frank RM, Cotter EJ, Strauss EJ, Gomoll AH, Cole BJ (2018) The utility of biologics, osteotomy, and cartilage restoration in the knee. J Am Acad Orthop Surg 26:e11-e25

34. Huebner K, Frank RM, Getgood A (2019) Ortho-biologics for osteoarthritis. Clin Sports Med 38:123-141

35. Le H, Xu W, Zhuang X, Chang F, Wang Y, Ding J (2020) Mesenchymal stem cells for cartilage regeneration. J Tissue Eng 11:2041731420943839

36. Marlovits S, Striessnig G, Resinger CT, Aldrian SM, Vecsei V, Imhof H et al (2004) Definition of pertinent parameters for the evaluation of articular cartilage repair tissue with high-resolution magnetic resonance imaging. Eur J Radiol 52:310-319

37. Jungmann PM, Welsch GH, Brittberg M, Trattnig S, Braun S, Imhoff AB et al (2017) Magnetic resonance imaging score and classification system (AMADEUS) for assessment of preoperative cartilage defect severity. Cartilage 8:272-282

38. Mehta S, Watson JT (2008) Platelet rich concentrate: basic science and current clinical applications. J Orthop Trauma 22:432-438

39. Ma J, Sun J, Guo W, Li Z, Wang B, Wang W (2017) The effect of platelet-rich plasma on reducing blood loss after total knee arthroplasty: a systematic review and meta-analysis. Medicine (Baltimore) 96:e7262

40. Everts PA, Devilee RJ, Brown Mahoney C, Eeftinck-Schattenkerk M, Box HA, Knape JT et al (2006) Platelet gel and fibrin sealant reduce allogeneic blood transfusions in total knee arthroplasty. Acta Anaesthesiol Scand 50:593-599

41. Gato-Calvo L, Magalhaes J, Ruiz-Romero C, Blanco FJ, Burguera EF (2019) Platelet-rich plasma in osteoarthritis treatment: review of current evidence. Ther Adv Chronic Dis 10:2040622319825567

42. Loia MC, Vanni S, Rosso F, Bonasia DE, Bruzzone M, Dettoni F et al (2016) High tibial osteotomy in varus knees: indications and limits. Joints 4:98-110

43. Fukui N, Yamane S, Ishida S, Tanaka K, Masuda R, Tanaka N et al (2010) Relationship between radiographic changes and symptoms or physical examination findings in subjects with symptomatic medial knee osteoarthritis: a three-year prospective study. BMC Musculoskelet Disord 11:269

44. Marcacci M, Zaffagnini S, Kon E, Marcheggiani Muccioli GM, Di Martino A, Di Matteo B et al (2013) Unicompartmental osteoarthritis: an integrated biomechanical and biological approach as alternative to metal resurfacing. Knee Surg Sports Traumatol Arthrosc 21:2509-2517

45. Chung YW, Yang HY, Kang SJ, Song EK, Seon JK (2021) Allogeneic umbilical cord blood-derived mesenchymal stem cells combined with high tibial osteotomy: a retrospective study on safety and early results. Int Orthop 45:481-488

46. Kim YS, Chung PK, Suh DS, Heo DB, Tak DH, Koh YG (2020) Implantation of mesenchymal stem cells in combination with allogenic cartilage improves cartilage regeneration and clinical outcomes in patients with concomitant high tibial osteotomy. Knee Surg Sports Traumatol Arthrosc 28:544-554

47. Song JS, Hong KT, Kim NM, Park HS, Choi NH (2020) Human umbilical cord blood-derived mesenchymal stem cell implantation for osteoarthritis of the knee. Arch Orthop Trauma Surg 140:503-509

\section{Publisher's Note}

Springer Nature remains neutral with regard to jurisdictional claims in published maps and institutional affiliations. 